\title{
Referenciales en la investigación jurídica
}

\author{
María LAge Cotelo \\ Universidad Nacional de Educación a Distancia (UNED) \\ mlagcot@gmail.com
}

Recibido: $19 / 07 / 2012$

Aceptado: 22/10/2012

\begin{abstract}
Resumen
La investigación jurídica está condicionada por factores ajenos a ella y que proceden primordialmente de las convicciones tanto sociales como morales o de conciencia del sujeto investigador. Ante el avance de la ciencia y la tendencia cada vez mayor a la diversidad social, la investigación jurídica necesita renovarse desde sus preceptos más básicos: los referenciales jurídicos en relación con la titularidad de derechos. Será objeto de análisis en el presente artículo la situación actual de la investigación jurídica y la pretensión de abogar por una libertad en la misma desde la libertad de conciencia aplicada a la titularidad de derechos concurrentes.
\end{abstract}

Palabras clave: referencia, investigación jurídica, subjetividad, norma, libertad de conciencia.

\section{References in the Legal Research}

\begin{abstract}
The investigation in the field of the legal system is determined by factors that don't belong to this matter. These factors come from the moral and social convictions of the investigator. Considering the scientific progress and the social diversity of our times, all the investigations in the legal system need to be updated from their roots. In our analysis, we'll study the actual situation of the investigation in the legal system and the aspirations of defending the liberty from the freedom of concience, put in practice over the existing rights.
\end{abstract}

Keywords: reference, legal research, subjectivity, rule, freedom of conscience.

\section{Referencia normalizada}

LAGE COTELO, María (2012): "Referenciales en la investigación jurídica". Estudios sobre el mensaje periodístico. Vol. 18, núm. especial noviembre, págs.: 561-568. Madrid, Servicio de Publicaciones de la Universidad Complutense.

Sumario: 1. Introducción. 2. Referenciales de la investigación jurídica. 3. Metodología y significado de la investigación jurídica en relación con los derechos; 3.1. Objetivación y subjetivación jurídica. Titularidad de derechos en investigación. 4. Conclusiones: el referente de la libertad de conciencia aplicado a la titularidad de derechos concurrentes. 5. Referencias bibliográficas.

\section{Introducción}

Cualquier planteamiento innovador dentro de un marco consolidado de derechos lleva implícito el problema de la referencia de ese derecho en relación con la titularidad de sujetos que ostentan ese mismo derecho. Desde una perspectiva cercana a la sociología del derecho, es fundamental la interacción dialéctica del lenguaje como fuente de conocimiento renovable al servicio tanto del propio conocimiento como herramienta hermenéutico-comunicativa.

Lo anterior, llevado a un ámbito estrictamente jurídico, positiviza el problema primario de la investigación jurídica o de la investigación jurídica como fuente aproximativa de una nueva realidad social del derecho.

Si ello lo relacionamos con la investigación y con la intencionalidad que el sujeto investigador tiene en su planteamiento, obviamente se incurre en la primacía de unos derechos subjetivos sobre otros, bien directamente bien indirectamente, pero siempre 
desde el matiz de la intencionalidad sobre el que descansa la expectativa de la objetivación del conocimiento de la ley.

Por todo ello es conveniente proponer un análisis revisionista de la situación actual de la investigación jurídica desde la concurrencia de la titularidad de derechos sobre un mismo objeto, objeto en este caso del análisis jurídico, pero que no limite la libertad investigadora.

\section{Referenciales de la investigación jurídica}

Investigar la manifestación externa de lo jurídico, no es teorizar sobre la manifestación externa de lo que es la norma, sea cual fuere su naturaleza, es rebatir la optimización de la norma en cuanto a la concurrencia de titulares de derechos a los que incluye esa norma: "el aspecto externo de una norma consiste en la regularidad de su cumplimiento y/o de la sanción de su incumplimiento. Lo decisivo es el comportamiento observable, aún cuando requiera ser interpretado" (Alexy,1994:22); y en efecto, requiere de su interpretación.

Dicho así, podría entenderse que de la manifestación de la norma simplemente nos interesa su aspecto externo, sin embargo, la manifestación interna de la norma, es el sujeto referencial en toda investigación jurídica: "el aspecto interno de una norma consiste en la motivación-de cualquier tipo que ella sea- de su cumplimiento y/o aplicación. Lo decisivo son las disposiciones psíquicas.”( Alexy,1994:23) En cierto sentido, el aspecto interno de una norma es la interpretación moral que el investigador jurídico realiza de ésta y sobre la que su derecho subjetivo entendido como manifestación externa del aspecto interno de la norma fáctica, se hace visible en la concreción de la investigación.

De la premisa anterior puede extraerse una conclusión ambigua respecto a la manifestación de la norma en cualquier sujeto, sea o no titular de derechos, esto es, un cierto paralelismo entre la norma dirigida al colectivo, la norma dirigida al individuo y la norma que el individuo asume e interpreta según el discernimiento de su moralidad intrínseca.

La creación del derecho sustentada en el imperativo de orden social carece de validez formal si tenemos en cuenta que la validez formal del mismo en una sociedad está sustentada primariamente en el nivel concordancia de la realidad con el nivel de introspección que se posea de la norma, así paulatinamente es el orden social el que conforma del derecho a pesar de que éste "ha ido creando un lenguaje técnico formado de conceptos que suelen partir del lenguaje común, pero que poco a poco van apartándose de él para convertirse en términos técnicos" (Cruz Parcero,2006:25) ; si ello se pone en consonancia con la titularidad, es decir con el sujeto normativo que pretende ser normado (y la atribución de unos derechos que éste posee por ser simplemente sujeto normativo antes de ser sujeto normado) se obtiene que en efecto "los derechos forman parte de un lenguaje normativo y consisten en razones para guiar y justificar nuestra conducta, para criticar o censurar, para exigir o demandar acciones y omisiones de otros, para imponer deberes, conferir facultades. El problema de caracterizar a los derechos como razones es que tenemos que responder a la pregunta sobre qué clase de razones morales son aquellas que reconocemos como derechos y 
ésta no es una tarea sencilla, aunque no tiene que ser una tarea imposible." (Cruz Parcero, 2007: 64)

En efecto, el problema de la subjetividad del propio sujeto que se enfrenta a la norma es el problema básico del derecho por el que se apela a esfera exclusiva e individualizada en la que tienen cabida, ya dentro de la propia norma, las concesiones dialécticas de su convicción a cerca de esa norma, y es que "desde que el derecho se comenzó a codificar, uno de los problemas que se le presentaron a los juristas era el de hacer compatibles, o al menos el de llamar la atención sobre problemas de inconsistencia o contradicción entre las normas o entre los comentarios existentes respecto de las normas" (Cruz Parcero, 2006: 26); esta posible inconsistencia o contradicción si pensamos la historia del derecho, no proviene simplemente del aspecto externo de la norma sino primordialmente de su aspecto interno que debe concordar con el aspecto intersubjetivo del sujeto titular de derechos en el que tenga cabida el nivel de asunción de la norma según su sistema. Pero por otra parte, la manifestación objetiva de la norma no permite que el titular del sujeto se exceda en cuanto a los derechos atribuidos que ésta le infiere para si, bien en su manifestación interna o en su manifestación externa. En el caso de la investigación sobre un derecho concreto, lo anterior, se traduce todavía en una complejidad mayor, puesto que el investigador en derecho presume una asunción personal de la propia norma sobre la que investiga y sobre la que quiere llegar a la conclusión que parte de la esfera exclusivamente subjetiva desde el aspecto formativo de lo que esa norma es para sí. Colisionan en este caso el derecho del sujeto que opera con la norma, el de la norma en su manifestación objetiva y subjetiva y el halo irreductible de la consideración individual de la norma y su maleabilidad. Podremos hablar entonces de un nuevo referencial en el tratamiento del hecho jurídico que va mucho más allá de la propia investigación, hablamos de derechos concurrentes desde diversos planos, conciliables o no, pero que coexisten en todas las esferas del derecho y que no escapan a la investigación.

La neutralidad en el caso de la investigación pasa por reconducirnos a la objetivación normativa a la que se alude en un momento anterior, sin embargo, no puede conseguirse en su totalidad siempre que lo que se pretenda del aspecto normativo del derecho sea su interpretación o su investigación pero por el contrario, no sucede o debiere suceder así en su aplicación puesto que "la interpretación de un texto jurídico jamás será aleatoria. Se trata de comprender que lidiamos con la sintaxis y que no podemos separarla de la semántica (por ejemplo, la vieja escisión hecho-norma) y tampoco entender la pragmática como "reserva técnico-hermenéutica" para resolver insuficiencias lógico-semánticas.” (Streck, 2012: 279). Sin embargo si nos ceñimos al contenido, al cierto utilitarismo en el sentido laxo del concepto en el que la norma es empleada, bien sea con fines de investigación, o simplemente procesales "si hay ocasiones en las que el derecho requiere que pasemos por alto el contenido proposicional de las disposiciones y establezcamos excepciones o límites en su alcance, ¿puede suceder otro tanto en relación al balance de razones subyacente a las mismas?; ¿puede el derecho obligarnos a cuestionar el balance de razones subyacente a una norma?" (Ródenas, 2012: 273), en el caso de que nos cuestionásemos las razones subyacentes a la norma, nos encontramos con una interpretación intersubjetiva en la que el usua- 
rio de la misma se hace eco de la libertad, llámese de conciencia desde sus propias creencias personales que subsumen la norma y la adaptan a su finalidad; la finalidad o el consecuencialismo en este caso no sería la única justificación loable, porque el mutante dinamismo de la convicción si tendría cabida en la aplicación reflexiva de la misma y debe ser respetada desde la propia norma objetivada por el sujeto.

\section{Metodología y significado de la investigación jurídica en relación con los derechos}

Al tratar de realizar una reflexión sobre la situación de la investigación jurídica, el punto de partida no es otro que la definición conforme a derecho de lo investigado, es decir, el tratamiento que se va a realizar conforme a derecho.

En este sentido y tal y como sucede en cualquier investigación, no es la asunción de la norma el ítem destacado y a tener en cuenta, sino la perspectiva desde la que lo jurídico va a ser revisada. Argumentar en este sentido no es más que una puesta en escena de lo que el jurista como investigador pretende revisar sobre la norma. En este caso "la argumentación jurídica consiste por una parte en un razonamiento de tipo deductivo, donde operan los principios y métodos de la lógica: a este paso del razonamiento jurídico se le ha llamado la justificación interna, y suele ser explicado como un razonamiento silogístico donde al menos una de las premisas es una norma. El otro paso es llamado la justificación externa, y consiste en establecer y justificar las premisas de las que parte la justificación interna. Un argumento jurídico contiene al menos una premisa normativa, una premisa fáctica y una conclusión normativa, de modo que normalmente los problemas que surgen respecto a dichas premisas son 1lamados, respectivamente, problemas normativos o fácticos." (Cruz Parcero, 2006: 3334) Traducido a la investigación actual, el argumento jurídico desde una perspectiva lógico-revisionista, tiene un patrón definido, sin embargo desde el punto de vista personal, la investigación pone de relieve no sólo la norma sino el grado de interiorización que se tiene de la misma conjugado a su vez con los elementos presentados con anterioridad: la moralidad del sujeto investigador, la moralidad presente en la sociedad y el grado de asunción del derecho como colectivo; pero asimismo es importante traer de nuevo a colación la libertad intersubjetiva que el sujeto plasma en la investigación, traducida a su vez ésta en la propia conciencia que determina lo jurídico desde un punto de vista exclusivamente subjetivo.

La relación del sujeto con la norma es evidente, y más en tanto que "la noción de existencia fáctica de una norma es descriptiva: si se dice que una norma está vigente en una determinada comunidad, se afirma un hecho. Por otra parte, se trata de un concepto susceptible de grados: la vigencia de una norma puede ser mayor o menor en cierto grupo, dependiendo ello de la medida en que sea cumplida, aplicada o aceptada por sus integrantes. Se trata, finalmente, de un concepto relativo: la vigencia se predica de una norma de cierta sociedad y en cierto tiempo" (Rodríguez, 2002: 48), al igual que el estudio del investigador jurídico. De ahí la importancia de que la consistencia del discurso jurídico sea lo más fiel a la conciencia de los sujetos de la época. Quizá no sea tan importante el aspecto innovador, sino más bien que el objeto teórico que es estático (Lage Cotelo, 2012) sea interpretado a través de la libertad de conciencia dado que "el método del derecho por no estar dirigido en primer lugar a "ex- 
plicar a partir de la necesidad" construye un objeto teórico (la prohibición o el permiso) que no "refleja" la realidad empírica" (De Lima Lopes, 2006: 51) sino la realidad teórica a favor del progreso social, cuya finalidad en el caso de la investigación jurídica es más que evidente; por ello, la crítica externa a la investigación jurídica es en esencia consecuencia de una colisión de libertades intersubjetivas que van más allá de la propia interpretación de la norma partiendo de la asunción de que "los conceptos de derecho orientados a la instauración normativa se encuentran, sobre todo, en el ámbito de la teoría analítica del derecho, es decir, en las proximidades de las corrientes de la teoría del derecho que, primordialmente, se ocupan del análisis lógico o conceptual de la práctica jurídica. Mientras que en los conceptos de derecho orientados a la eficacia predomina la perspectiva del observador, en los conceptos de derecho orientados a la instauración normativa figura, en primer plano, la perspectiva del participante" (Alexy, 1994: 24), y por participante puede entenderse el aspecto social que con mesura incide indirectamente en la creación de derecho, no simplemente norma sino el concepto totalitario que desde una perspectiva social es la que adecua su problemática al derecho positivo.

\subsection{Objetivación y subjetivación jurídica. Titularidad de derechos en investigación}

Mucho es lo que se ha teorizado en relación con la vertiente objetiva y subjetiva de los derechos, así como muchas han sido las definiciones que a lo largo de la historia han aparecido de los mismos.

Primordialmente lo que persiguen dichas definiciones es algo menos concreto que una calificación, persiguen un halo de libertad en el que el sujeto tenga autonomía respeto a la objetivación o subjetivación de la norma, y esta "libertad, pues, viene creada, por el fin que persigue el individuo: como sucede casi siempre, la causa última o final es la que justifica la conducta antecedente que quiere abrirse paso." (Alexy, 1994: 24) En la investigación jurídica también se debe hablar de la objetivación y de la subjetivación del derecho, pero desde la propia subjetivación que comporta toda investigación. Si nos limitamos a conferir simplemente la subjetividad de la investigación a la objetivación o subjetivación de o del derecho concreto sobre el que se teoriza, encontramos la limitación proveniente de una esfera externa a la investigación, esto es "el ejercicio de los derechos subjetivos tiene unas limitaciones, que dará lugar a una actuación negativa del orden público, que prohibirá o excluirá determinadas conductas. A su vez veremos una vertiente positiva, en la que el término límite debe ser entendido como garantista porque marcará los ámbitos protegidos del orden público, entendidos como ámbitos del libre ejercicio de las libertades", sin embargo, "es lógico que al llamar "derechos subjetivos" a todas las situaciones de poder reconocidas por las normas jurídicas" (Carpintero,1993: 269) y siendo reiterativos, la investigación jurídica no escapa a la norma, ni moral ni legal del contexto socio-cultural en el que se encuentra inserta, es decir, la vigencia temporal que coincide de modo exacto entre la investigación y la normativa aplicable. Pero el orden socio-cultural actual, en el que tiene cabida el multiculturalismo y la coexistencia de diversidad de planteamientos en atención a la convivencia de ese multiculturalismo, tiene que superar los planteamientos que se consideran invasores y no estigmatizar la investigación novedosa 
en pro del presente; es decir, con la experiencia histórica se ha debido conseguir la superación del viejo esquematismo coercitivo que limita la validez de la norma moral exclusivamente con la relación de ésta y el medio, si bien se entiende que "las normas de una sociedad se determinan en función de principios de justicia abstractos, pero también en función de su inscripción en un contexto cultural propio (su demografía, su historia, etc.)" (Elósegui Itxaso, 2009:43) , pero no son supuestos tasados o no debieren presentarse como tales.

El contexto social de la reflexión sobre la norma, cobra más sentido en el caso de que no exista una regulación expresa (o la existencia de un vacío legal que sea proclive a la introducción de nuevas directrices propiciadas por la reflexión crítica) por ello, "la invasión de la filosofía por el lenguaje, al proporcionar la superación del esquema sujeto-objeto, coloca el lenguaje como condición de posibilidad, siendo vedado utilizarla-bajo pena de una paradoja de cuño paradigmático-como un instrumento, finalmente, como una tercera cosa que proporcione la hipostasiación de discursos (en el caso, de discursos fundamentados previamente, contrafácticos) y una procedimentalización argumentativa, que deja en segundo plano la aspiración final de la norma: la aplicación. En síntesis, la superación del esquema sujeto-objeto, elevado como soporte para la sustitución de la razón práctica justamente en ella sostenida, es incompatible con discursos contrafácticos, porque éstos, en vez de proporcionar la síntesis intersubjetiva de la que emerge el sentido (por que los sentidos ya no están en las cosas ni en la mente), promueven, queramos o no, una especie de retorno al esquema deductivista, una vez que, escindidos, los discursos de fundamentación, por ser previos, tienen la función de servir, contrafácticamente, de categorías para el encuadramiento (la adecuación) de las situaciones concretas, que, así, pueden ser vistas como hechos (facticidad) "aún sin sentido", a la espera del debido acoplamiento." (Streck, 2012: 47) La presencia por tanto de indicadores negativos en relación con la aceptación externa es un dato a tener en cuenta. Sucede que las investigaciones lejos de considerarse libres, gozan de unas ciertas restricciones, en las que se conjuga perfectamente una lucha entre el derecho subjetivo y el derecho objetivo de la relación jurídica analizada y, de la relación jurídica con el propio sujeto investigador, que lejos de ser neutral coexiste con su contexto histórico, y en este sentido desde el punto de vista jurídico-social "la interpretación del orden público va inexorablemente unido a las mentalidades culturales, también en sentido positivo. [...] La cultura es cambiante y la cultura jurídica también" (Elósegui Itxaso, 2009: 50-51), por lo tanto, "el estudio estático de una estructura o de una institución social en un momento determinado consiste necesariamente en una investigación sobre variables interconectadas, internas o externas. Puesto que la investigación es, precisamente acción y es, en todo caso, cuantificable en razón del tiempo mayor o menor en el que se desarrolla, se deduce que en principio el estudio del cambio supone la comparación entre campos de variación cronológica." (Ferrari, 2001: 292) Así, el problema temporal en derecho es quizá mucho más arraigado que en otras disciplinas; este hecho tiene que ser entendido desde dos puntos de vista distintos: desde la vigencia y temporalidad de la ley y desde el constante cambio socio-cultural, lo que se puede resumir como que "la diferencia (que es ontológica) entre texto y norma (sentido enunciativo del texto, o sea, el modo como 
lo podemos describir fenomenológicamente) tiene lugar en la incidencia del tiempo" (Streck, 2012: 265) al estar acotado el derecho positivo por la propia positivación de la norma, "es decir, los derechos subjetivos se ejercen dentro de los límites propios que les corresponden. Deben ser respetados y acatados por los distintos sujetos jurídicos y protegidos por el ordenamiento [...] Son ámbitos del libre ejercicio del derecho, es decir, entran en el campo del ejercicio legítimo de los derechos de libertad, entre ellos el de libertad cultural" (Elósegui Itxaso, 2009: 54), idea principal sin duda ante la titularidad de derechos y la inserción de los mismos en el contexto socio-cultural en el que deriva su aplicabilidad. El problema de la libertad cultural, en cierto sentido no deja de ser un reflejo de indicador negativo de la aceptación externa respecto a la libertad vital del sujeto; bien se presente éste como sujeto referencial bien sea el indicador externo el que limite la propia libertad vital.

\section{Conclusiones: el referente de la libertad de conciencia aplicado a la titularidad de derechos concurrentes}

El compromiso de la investigación, y más si cabe en la investigación jurídica (como exponente de derechos tratados a expensas de la finalidad que cumplen en relación con su nivel de beneficio o utilidad social) aporta o puede aportar datos significativos que propiciarían grandes avances en todos sus ámbitos que directamente pudieren repercutir a través del derecho positivo en la sociedad.

Estos paulatinos cambios parten usualmente de dilemas morales sustentados en la esfera intersubjetiva del sujeto investigador y se materializan en el objeto investigado. En este sentido, la máxima del investigador jurídico debe tender a la no coerción de la libertad de su planteamiento jurídico acorde o no a derecho. Con ello se quiere apelar a la esfera de libertad que todo investigador debe poseer para poder avanzar sin las (por otra parte lógicas) limitaciones que existen en cuanto al derecho positivo, en muchos casos restrictivo para la finalidad de la propia investigación. Si todo ello, lo traducimos en términos de moralidad y libertad de conciencia, premisas por otra parte básicas, desde las que el sujeto investiga, se hace complejo delimitar la prevalencia en términos de libertad de conciencia o moralidad entendida desde la concurrencia de planteamientos divergentes y más si cabe, si estos planteamientos proceden no del conflicto moral que puede suscitar una norma, sino de la reflexión legal sobre la misma en la que la moralidad y la libertad de conciencia se encuentran inevitablemente presentes.

En una relación de la vertiente ética con la libertad de conciencia como elementos constitutivos de una titularidad propia y privada del sujeto, es ineludible su tratamiento o referente constitucional como elemento influyente y genérico. Ante ello, "la determinación de una ética común para todos los ciudadanos parece difícilmente conciliable con el reconocimiento universal de la libertad y autonomía de la conciencia, como parámetro ético de la actuación de cada individuo. La separación entre moral y Derecho ha contribuido a alejar del ámbito social y público cualquier idea de una ética común vinculante para todos los ciudadanos y, por tanto, como posible fundamento de las normas jurídicas." (Souto Paz y Souto Galván, 2011: 153) Y esta escisión entre moral y derecho, entre una posible colisión de la moral particular con la moral pública derivada de la norma no debe ser perdida de vista por el visionario investigador que 
con su reflexión crítica anhela la consecución de un cambio, porque si con la investigación jurídica no se pretende una consecución de un derecho no consolidado en el sistema normativo, estamos ante una teorización o reformulación de la positivación del derecho, pero que consecuentemente no será de utilidad para el progreso social, que en última instancia, es a lo que debe tender toda investigación, sea o no jurídica.

\section{Referencias bibliográficas}

ALEXY, Robert (1994): El concepto y la validez del derecho. Barcelona, Gedisa

CARPINTERO BENÍTEZ, Francisco. (1993): Derecho y ontología jurídica. Madrid, Actas.

CRUZ PARCERO, Juan Antonio (2006): Los métodos para los juristas. En Courthis, Ch. Ed. Observar la ley. Madrid, Trotta.

CRUZ PARCERO, Juan Antonio (2007): El lenguaje de los derechos, ensayo para una teoría estructural de los derechos. Madrid, Trotta.

DE LIMA LOPES, José Reinaldo (2006): Regla y compás, o metodología para un trabajo jurídico sensato. En COURTHIS, Ch. (Ed.): Observar la ley. Madrid, Trotta.

ELÓSEGUI ITXASO, María (2009): Derechos Humanos y pluralismo cultural. Madrid, Iusel.

FERRARI, Vincenzo (2001): Acción jurídica y sistema normativo. Madrid, Dykinson.

LAGE COTELO, María (2012): La docencia teórica en la investigación: el desafio de la maestría persuasiva. http://www.fpce.up.pt/ciie/cidu/publicacoes /livro_de_textos.zip

RÓDENAS, Ángeles (2012): Los intersticios del derecho, indeterminación, validez y positivismo jurídico. Madrid, Marcial Pons

RODRÍGUEZ, Jorge L. (2002): Lógica de los sistemas jurídicos. Madrid, Centro de Estudios Políticos y Constitucionales.

STRECK, Lenio Luiz (2012): Verdad y Consenso. De la posibilidad a la necesidad de respuestas correctas en Derecho. Buenos Aires, B de F.

SOUTO PAZ, José Antonio y SOUTO GALVÁN, Clara (2011): El derecho de libertad de creencias. Madrid, Marcial Pons.

\section{María LAGE COTELO}

Investigadora Facultad de Derecho UNED

mlagcot@gmail.com

Máster Universitario Derechos Fundamentales

Especialista en Derecho de Extranjería y Derecho de Familia

Especialista en Cultura y Modelos Culturales

Experto Universitario en Derecho Procesal y Tráfico jurídico 